\title{
E-Health Biosensor Platform fOR NON- INVASIVE HEALTH MONITORING FOR THE ELDERLY IN LOW RESOURCE SETTING
}

\author{
Okuonzi John ${ }^{1}$ and Callixte Yadufashije ${ }^{2}$ \\ ${ }^{1}$ Department of Electrical and Electronic Engineering, Kyambogo University, Uganda \\ ${ }^{2}$ Department of Biomedical Laboratory sciences, (INES-Ruhengeri), Rwanda
}

\begin{abstract}
New technologies in the field of tele-health using biosensor systems for non-invasive vital signs monitoring of patients, especially elderly people who need long-term care, and marginalized areas with hard to reach health care services are emerging. A study involving a self-care approach within the cardiac domain, where late detection increases the likelihood of patient disability or of premature death is proposed. In the study the application of e-health biosensors platform in medical services is experimented. The study resulted into the synthesis of vital signs from various body positions with biosensors that does not require a full coupled system. A model for the prevention of cardiovascular disease management based on noninvasive personal health monitoring systems with easy access for everybody, at any time or location is designed. A personal vital sign system such as ECG sensor which contain the functionality, allows recording anywhere and at any time a diagnostic quality ECG and analyzing it "on-board" by comparing it to a reference ECG, is modelled. The model called Mobile Health for the Elderly Persons (MOHELP) which relies on with application in estimation and control of boolean processes based on noisy and incomplete measurements is designed. This enabled a reliable recommendation from a digital artificial intelligence-based diagnosis, which can support an elderly person to take timely and correct decisions upon his (her) health status. In a case of urgency, the assistant puts the elderly person in a contact with healthcare providers. The signal pattern sensitivity related to sensors placement is one of the issues this study addressed using e-sensor platform. Sensors displacement errors have a direct impact on the medical diagnosis, especially if the diagnostic procedure is automated. The study resulted into the formulation of a methodology for e-Health Sensor Platform, in software architecture terms, that permits use of system biosensors to adapt to the user-specific context for self-healthcare.
\end{abstract}

\section{KEYWORDS}

e-Health, Biosensor, eHealth sensor platform, elderly persons, vital signs, ECG

\section{INTRODUCTION}

In the East African regions, particularly in Uganda, the number of the elderly (over 60 years old) is on the increase. The number has increased from 1.3 million in 2010 and is expected to reach nearly 2.5 billion by 2025 (WHO, 2013). With this trend of growth, it is projected that elderly people aged over 60 in Uganda the was nearly 1.3 million in 2013 and will reach 4 million by 2025 (2013). Furthermore, Uganda's population is aging fast, with an elderly population of 34\% of total population (WHO, 2015). Accompanying this phenomenon of the increasing number of the elderly is the exponentially increasing healthcare costs for long-term care and hospitalization due to the rising number of the elderly, yet they marginalized economically in least developed countries. The elderly predicament is compounded by the fact that most of the health care facilities in developing countries are dysfunctional or far or difficult to access in case of 
emergencies. Ailment brought about by aging such as chronic illnesses bring about needless longterm hospitalization with huge costs of healthcare. Therefore, it is essential for elderly people to take safeguards against likely long-lasting and acute ailments.

With the motivation to address challenges associated health care issues of marginalized elderly persons aged 60 above in a developing country like Uganda, a study of the application of biosensors for non-invasive monitoring of a chronic condition was proposed. The proposed study carried from 2014 to 2016 underscored the telecare service development with e-health sensor platform for non-invasive monitoring. The need of elderly today is to lengthen lifetime, dignity with preserved quality of life and ability to actively participate in self-health management. A noninvasive self-care approach allowing the diagnosis, management and monitoring of health conditions of the elderly persons and aiming at effective detection and treatment of any problem before it necessitates complex and costly emergency interventions becomes particularly attractive in this context. These current demographic and cultural changes demand the traditional medicine, which is mainly focused on disease treatment in centralized healthcare units by professionals, to make necessary reforms to get along with new expectations of society and to ensure the quality of service in these changed conditions (Asta, 2011).

When an elderly person takes an active role in their health, which is supported by physician or pharmacist roles and makes constant check-ups when he (her) thinks they are needed, the feeling of dignity is experienced. This situation prolongs life and sets in a smart self-care environment, which could be achieved by the use of a personal smart device e-Health Sensor Platform) capable to record and analyze a patient specific vital sign such as the electrocardiogram (ECG). The ECG recording remains indeed the most important, independent, objective and non-invasive source of information for the clinical evaluation of patients with chest pain (Lee, 2000; Garvey, 2006), and/or heart rhythm abnormalities (Zipes, 2006). The personal device needs to have at least two principal elements: 1) the smart agent (monitor), which may be a separate wearable device, or attached to the body to create a body area network (BAN), a mobile phone, a watch, or even installed into an ordinary personal computer and serves for signal recording, storing, processing and analysis, and 2) a sensor-system, which must be easily, though reliably and conveniently placed on an individual in their appropriate location by a non-specialist citizen himself, in order to capture the heart's electrical signal. A personal vital sign system such as ECG sensor which contain the functionality, allows recording anywhere and at any time a diagnostic quality ECG and analysing it "on-board" by comparing it to a reference ECG, if possible. In addition, it is capable of making a reliable recommendation from a digital artificial intelligence-based diagnosis, which can enable an elderly person to take timely and correct decisions upon his (her) health status. In a case of urgency, the assistant shall put the elderly person in a contact with healthcare providers.

General Electrics (GE) introduced the Personal Health Systems (PHS) in 1990s to describe a new type of health care delivery process, which puts the patient at the centre of it and enables them to take timely decisions with the help of new tools that ensure pervasive self-care. Starting from then new tools capable to record one or several bio-signals (pulse, body temperature, respiration rate, electrocardiogram and others) in home-based conditions were developed. These novel systems were aiming at convenient and robust patients monitoring in their homes while they are performing their daily activities, trying to not interfere significantly with their comfort or lifestyles. Quickly wearable sensor technologies took there a pivotal role. Current research projects are focusing on four groups of wearable monitoring platforms: (i) "Holter-type" systems with standard sensor design and locations, (ii) Body-worn sensor patches, (iii) Body-worn bands 
and harnesses and (iv) "Smart garments" for long-term application (McAdams, 2011). However, all these already established approaches have various limitations, for an on-demand use of the corresponding sensor-systems by every citizen, especially in self-care situations. For instance, they need a constant professional supervision or a prolonged continuous wearing and so they do not ensure the independence.

In case of occasional monitoring, a potential clinical need is to have at the user's disposal a small, portable system, or integrated into a mobile phone or a watch, which goes together with a special sensor-system, a kind of a bib or an apron, that is easily placed on the thorax to capture the heart electrical signal, easily transported and wear on and off in any place. The system must be compliant with the body surface map configuration and supports a quick and discrete recording of vital signs. It also provides an initial diagnosis leading the user to make a rapid and evidencebased decision about their health status. This direct feedback or the possibility to send information to a remote monitoring station in case of an emergency is a key target of the e-Health Sensor Platform Project (Cooking Hacks, 2012-14). The project outcome is a prototype of 10 sensors including a Personal ECG Monitor (PEM), which includes a reduced-electrodes set that allows 10 seconds recording of three pseudo-orthogonal ECG leads I, II and V2 according to the Mason-Likar system. It also embeds algorithms allowing the reconstruction of standard 12-lead ECGs and a risk stratification based on signals analysis. However, as it has been widely reported, the diagnostic accuracy of the ECG and thus the success of the PEM and of similar systems depends on the user ability to apply sensors in the correct anatomical positions. This task requires a specific knowledge and time to find accurate electrodes' locations, and according to several studies remains problematic even for skillful professionals (Rajaganeshan, 2008, McCann, 2007, Sejersen, 2006). There is therefore need for high computational requirements to achieve ECG mining placed on the body. There is often high level of noise generation by the leads placed on a patient body. Models to with application in estimation and control of Boolean processes based on noisy and incomplete measurements have been proposed in (Mahdi \& Ulisses, 2017). Ghoreishi, et. Al (2017) also proposed a novel uncertainty propagation approach for multidisciplinary systems with feedback couplings, model discrepancy, and parametric uncertainty. Due to the dynamic nature of ECG data to be collected from a patient body, there is need to accommodate both static and non-static vital signals generated. Shuilian Xie., et. al. (2018) proposed a method of Linear Discriminant Analysis (LDA) classification rule for nonstationary data, using a linear-Gaussian state space model. Signals generated from different system that are coupled together such as the e-Health sensor platform and vital signs from electric signal from a human body pose key challenges of discrepancy, and parametric uncertainty of signs read. Ghoreishi and Allaire (2017) proposed a method of Adaptive Uncertainty Propagation for Coupled Multidisciplinary (AUPCM) to reduce the state of discrepancy and uncertainty.

The signal pattern sensitivity related to sensors placement is one of the issues this thesis addresses using e-sensor platform. Sensors displacement errors have a direct impact on the medical diagnosis, especially if the diagnostic procedure is automated (Schijvenaars, 1997). It is crucial to propose personalized and automatic electrode placement solutions in order to overcome the intersubject and intra-subject variability and to ensure the records quality, thus an accurate and faithful diagnosis. Current e-Health sensor platforms and devices intended to fulfil Personal Health Systems scenarios would greatly benefit of this achievement, which would give them a strong input to pass beyond the prototype phase towards clinical trials. Hence the study addressed mainly the: "How can we provide non-invasive personalized monitoring assistance for the elderly 
persons pervasively with limited human expertise with the use of eHSP parameters obtained from biosensors?"

\subsection{Technological Context Of Ehealth Biosensor Platform Application In Health CARE}

Ubiquitous computing in electronic Health or eHealth is often mentioned in the context of improving health care with its promise of better patient care and lower costs (GSMA, 2013). Certainly, ubiquitous systems are the cornerstone of telemedicine, vital signs monitoring, and ambient assisted living, which according to technology forecasts will be common lifestyle elements in the future society (Micheal, 2014). The eHealth sensor platform and MySignals allows the measurement of more than 20 biometric parameters such as pulse, breath rate, oxygen in blood, electrocardiogram signals, blood pressure, muscle electromyography signals, glucose levels, galvanic skin response, lung capacity, snore waves, patient position, airflow and body scale parameters (weight, bone mass, body fat, muscle mass, body water, visceral fat, Basal Metabolic Rate and Body Mass Index) (Cooking Hacks, 2015).

This sought vision is strongly supported by the global Information Society Technologies, Cooking Hacks and GSMA programs, which in years 2006 to 2018 set a special focus on early illness detection and encourage non-invasive health monitoring systems with flexible access for everybody, at any time and any location. GSMA and Cooking Hacks approach is crucial within the domain of healthcare applications such as cardiac-care, where the ischemic heart disease is classified worldwide to be the first cause leading to death and is among top six causes of burden of disease (Mathers, 2008; GSMA, 2013). Ease of detection and continuous monitoring through vital medical signs to enable monitoring is the basis of this study.

\subsection{Vital Medical Signs And Conditions For Monitoring}

A medical condition that is common among the elderly and can lead to cardiac failure is Ischemia. Ischemia is a condition when blood flow restriction occurs (Pantley, 1884). This condition is common among the elderly related to blood vessels and causes the damage or the dysfunction of the insufficiently irrigated body tissue often leading to stroke (Debra, 2015). According to Macfarlane (2010) Cardiac ischemia is caused by the insufficiency of blood flow and so the lack of oxygen supply to the heart muscle. In the elderly persons the Coronary Artery Disease (CAD) causing cardiac ischemia is the most common type of heart disease.

The CAD occurs in the elderly occur when the arteries that supply blood to the heart muscle (the coronary arteries) become hardened and narrowed due to build-up of material called plaque on their inner walls. The plaque, known as atherosclerosis, increases inside of coronary arteries and starts blocking the blood flow so reducing the much-needed oxygen supply to the heart muscle. The Pulsi oximeter $\left(\mathrm{SPO}_{2}\right)$ in the Mysignal sensor can be used to measure and monitor the oxygen level in the blood.

With time in the elderly persons CAD weakens the heart muscle and contributes to heart failure, which may reveal itself through angina, various arrhythmias or even Acute Myocardial Infarction (AMI), commonly known as a heart attack. Angina (angina pectoris) is a medical term for a chest pain, which happens when the heart muscle getting not enough oxygen produces a by-product called lactic acid that builds up in the muscle and causes the pain. This pain is usually described by a bunch of symptoms, such as a discomfort, heaviness, pressure, fullness, aching, burning, or painful feeling in the chest area (but may also be felt in the shoulders, arms, neck, throat, jaw, or 
back), and unfortunately, is often mistaken with indigestion so disturbing acute infarction diagnosis and thus an early treatment. The e-Health sensor platform can be used to measure all these vital signs. Common symptoms of heart failure in the elderly include; shortness of breath, cough, swelling of feet/abdomen, weight gain, and others most often begin slowly and are hardly noticeable without a careful examination (GSMA, 2010), hence the need for monitoring.

WHO (2012) reports that some elderly persons have ischemic episodes without knowing about them. This silent ischemia can lead to heart attacks that occur without warning, and sometimes even without a pain, hence leading to fatalities (Debra, 2015). In McLean (2011), it is stated that the heart failure does not mean that the heart has stopped or is about to stop as it might be thought. It means that the heart is failing to pump blood the way that it should to meet body needs, including needs of itself. When the heart does not get enough blood to operate properly and to respond to the body demands of more oxygen and nutrition, especially when a body strain appears, the electrical system responsible for the regular and coordinated contraction of the heart muscle might be affected as well and might lead to an irregular heart rhythm - arrhythmia. This can be a reversible situation - remove the strain and things return to normal, or irreversible - the damage permanently affects the electrical heart system causing cardiac Arrhythmias. The electrical impulses may happen too fast, too slow, or erratically, causing the heart to beat too fast, too slow, too early or irregularly and so disturbing the effective blood pumping. Most arrhythmias that cause short occasional abnormal awareness of heart beat (palpitations) even being merely annoying are more or less harmless. Others, though, lasting longer or occurring more often, may have a serious impact on the body as due to the abnormal heart activity the blood flow is significantly reduced (risking to form thrombus as well) and results in continuous oxygen and nutrition insufficiency (or even block), which leads to the damage of organs. This becomes life threatening if the brain, the heart or lungs are affected. That is exactly how a heart attack happens. A blood clot develops at the site of plaque in a coronary artery and suddenly cuts off most or all blood supply to that part of the heart muscle. Cells in the heart muscle begin to die if they do not receive enough oxygen-rich blood, causing a permanent damage to the heart muscle. If this situation is left untreated for a sufficient period of time it can cause a death -of heart muscle tissue (myocardium). According to Cooking Hacks, the e-Health sensor platform can be used to diagnose and monitor these conditions pervasively using the embedded sensors.

The report by Markey (2004) reveal that more than 70\% elderly persons, who suffered from a stroke, die or become dependent on others for help with their everyday living. This indicates prevention and monitoring is a high priority strategy. Anderson (2009) noted that, patients would have great chances to survive if the treatment would be delivered on time - "the earlier treatment is given the better", in the developing countries "almost $80 \%$ of cardiac deaths occur outside the hospital". Clinical studies done in Uganda show that the most crucial element affecting the survival of patients having a heart attack is how quickly the arteries of the heart are re-opened using a thrombolytic medication or applying the surgery called Percutaneous Coronary Intervention (PCI), known as coronary angioplasty (Ezati, 2008).

In order to respond immediately, the symptoms shall be recognized and adequate actions have to be taken as early as possible. Elderly persons in developing countries usually do not to recognize myocardial infarction, however in real situations symptoms are often interpreted incorrectly and only a small amelioration shortly after the course has been reported (Ezati, 2008). Then, the objective of eHealth platform and especially for personalized Health management, is to equip elderly persons with more efficient and responsive tools that would assist in decision making enabling citizens themselves to constantly undertake the primary health provision so reducing the 
time between the detection of first ischemia symptoms and the appropriate treatment (GSMA, 2014; Cooking Hacks, 2015).

Pollen (2009) observed that characteristically, elderly persons with acute chest pain and/or heart rhythm abnormalities potentially of ischemic origin presenting at emergency departments situations are assessed with three principal tools: "the history of the event, the 12-lead electrocardiogram (ECG), and cardiac enzymes and other serum makers of myocardial injury". Fayn, (2010) also noted three tools on the ECG is suitable to sustain daily non-invasive self-care routine performed by the elderly persons, as it is a non-obtrusive source of instant and objective information.

Sejersten (2010) explained that the morphology of ECG recording (even of 10 seconds long) contains considerable diagnostic information about the heart's activity and so a signal analysis can provide evaluation that supports the decision-making. He stated "ST-segment deviation will evolve in ECG leads oriented along the axis of the current of epicardial injury after an acute coronary occlusion occurs, and thus, ST-segment analysis has become an important non-invasive tool for diagnosing acute myocardial infarction and for deciding on and evaluating the efficacy of therapy."

The Cooking Hacks (2012) and Rubel (2005) affirmed the potentially helpful tool would be a small smart personal device having the capability to record discretely anywhere and at any time a diagnostic quality of a vital sign such as ECG and fitting into our life as naturally as blood pressure monitors have recently done. This device with sensors should have an embedded intelligence that is capable of analyzing a vital sign "on-board" and of comparing it with a reference vital sign. Likewise, it should be capable of making a diagnostic recommendation and, when needed, of putting a patient in a contact with the appropriate medical service. The objective of this study is to support the success of this challenging but potentially very rewarding aim based on the e-Health Sensor platform by elucidating important points of consistent techniques selection and enhancing already available calculations in order to meet pervasive-care requirements for the elderly. Indeed, the experimental use of ECG in such personalized tools has be based tested with reflections of the whole system, which can be constructed from a variety of different, though, related methods of vital sign recording and processing.

\section{MeTHODOLOGY}

\subsection{Experimental Design, Materials And Methods}

\section{STUdy Population}

Eight patients with a heart indication aged at 60 years and over attending the private clinic at a Kampala based General Hospital in Uganda were sampled for this study. The details of the hospital is not revealed to ensure privacy of information. Written consent was obtained before the participation in the study. There test ECG indications was to provide reference points for the study.

\subsubsection{STUDY PROCEDURE}

Simultaneous 12-lead ECG recording using the Mason-Likar 12-lead electrode arrangement and three study eHSP surface electrodes placed in the conventional MS400 electrode positions were recorded and simulated for three minutes. The reference ECGs were recorded with the participant 
in a horizontal position with the head and shoulders raised to approximately $30^{\circ}$. The electrodes used were single patient use ECG electrodes connected to the eHSP system with a shielded carbon cable $(1.5 \mathrm{~m})$, microcoax to unipolar snap. ECGs were recorded using a eHSP multichannel controller with signal recorder attached to a laptop running MATLAB software program. The sampling rate was set at $2400 \mathrm{~Hz}$. ECGs were stored as a binary encoding. The system setup of the eHSP is shown in Figure 11. In the setup of the system, the monitored patient was represented by a patient simulator with parameters set to match the indications on elderly sampled patients (to accepts instructions through a smart phone or tablet). The approach is a semiexperimental.

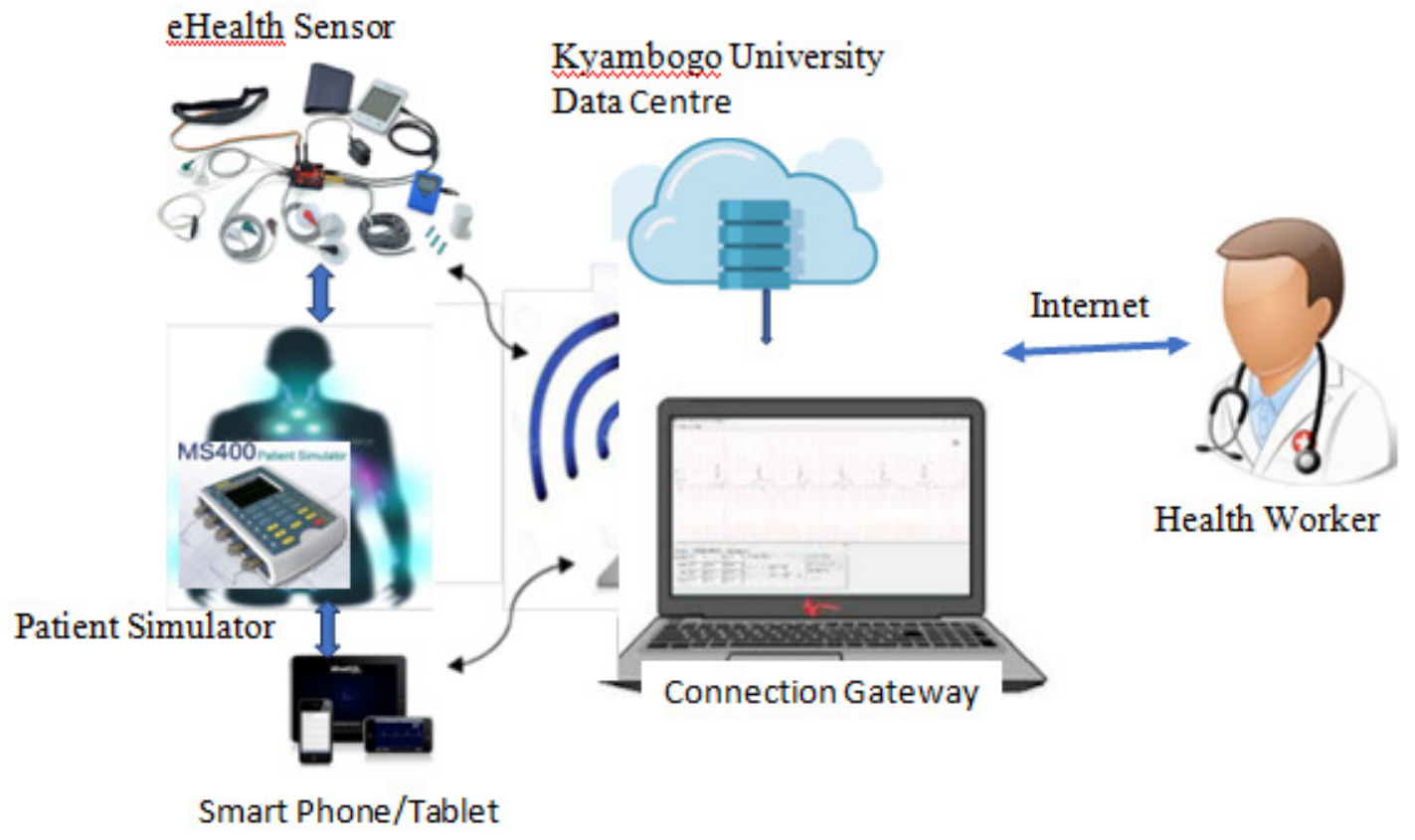

Figure 1: eHealth Sensor Platform setup

A semi-experimental test was arranged using the eHealth sensor kit with the upgraded MATLAB algorithm connected to a patient simulator as a reference. To evaluate the performance of the eHealth Sensor Shield V2.0 with Arduino Uno platform a patient simulator was linked to it in a real-time test using. The experiments were run on a Ubuntu 64-bit workstation with HP 21 inch Touch All-in-One that includes $4 \mathrm{GHz} 4$ th Generation Intel ${ }^{\circledR}$ Core ${ }^{\mathrm{TM}}$ i7 processor, 8GB RAM with all background processes switched off. The system is installed with Wireshark 2.0.5 (Cooking Hacks, 2014) to analyse the real-time traffic generated by the eHSP. The EHSP with the help of sensors gets the biometric data from the patient simulator (elderly patients) and is transferred to the server for storage or in real-time to the mobile device of the approved health worker for analysis. The eHSP wad set up to sense up to ten different kind biometrics, as glucometer, body temperature, electrocardiogram (ECG), patient position (accelerometer), oxygen in blood (SPO2), galvanic skin response (GSR-sweating), blood pressure (sphygmomanometer), air flow (breathing), and muscle/electromyography (EMG) sensor. In the study experimentation the ECG sensor was only enabled. The XBee radio module was fitted in the communication socket for communication. The data was transferred securely from this EHSP to the gateway and forwarded from the gateway to the database server (Kyambogo University Data Center). 


\subsubsection{SELECTION OF REPRESENTATIVE BEAT FOR ANALYSIS}

The representative QRST complexes that were compared were average beats over each of the recorded ECG leads generated in the following way: QRS complexes were sensed using an Rwave amplitude adaptively decaying sense threshold and any over-sensed beats were manually removed. The QRS fiducial point simulated for each patient was aligned for the three leads by selecting the median QRS peak sample in time with the first beat in each lead. The starting template was designated to begin 100 milliseconds (ms) prior to the sensed fiducial points and end $450 \mathrm{~ms}$ after the sensed fiducial point.

\subsubsection{Generation Of A Signal Averaged Beat}

The correlation coefficient of the template versus each of the following beats was calculated. Initially, only beats with a correlation $>0.80$ were included in the average. Then, the correlation coefficient was adjusted per lead between 0.5 and 0.9 to ensure the numbers of beats in each lead were similar. In addition, the difference in area between the template and the following beats were assessed for similarity and following beats were excluded if the area was not similar. The resulting signal averaged QRST complex from each simulated patient and lead was used as the "representative" beat for which the transformation coefficients were computed.

\subsubsection{Generation OF COEFFICIENTS, CONVERSION MATRIX AND DERIVED ECGS}

The transformation coefficients were derived using the least squared difference approach, in which they were optimized for minimum root mean squared (RMS) difference between measured and derived vectors when applied to the training dataset. The optimization was performed in MATLAB (MathWorks, 2014) using the optimization toolbox function 'fmincon' which is a constrained nonlinear optimization method using the interior-point algorithm. This optimization algorithm is designed to efficiently determine the transformation matrix from the horizontal and vertical leads of the eHSP to an 8-lead ECG that minimizes the RMSD between measured and computed 8-lead ECGs over all patients and leads.

The following model was created for the matrix calculation from two independent eHSP vectors to an 8-lead ECG:

$$
\begin{aligned}
& \text { leadI }=\alpha 1 \times \mathrm{H}+\beta 1 \times \mathrm{V} \\
& \text { leadII }=\alpha 2 \times \mathrm{H}+\beta 2 \times \mathrm{V} \\
& \text { lead III }=\alpha 3 \times \mathrm{H}+\beta 3 \times \mathrm{V} \\
& \text { lead aVR }=\alpha 4 \times \mathrm{H}+\beta 4 \times \mathrm{V} \\
& \text { lead aVL }=\alpha 5 \times \mathrm{H}+\beta 5 \times \mathrm{V} \\
& \text { lead aVF }=\alpha 6 \times \mathrm{H}+\beta 6 \times \mathrm{V} \\
& \text { leadV1 }=\alpha 7 \times \mathrm{H}+\beta 7 \times \mathrm{V} \\
& \text { leadV2 }=\alpha 8 \times \mathrm{H}+\beta 8 \times \mathrm{V} \\
& \text { leadV3 }=\alpha 9 \times \mathrm{H}+\beta 9 \times \mathrm{V} \\
& \text { leadV4 }=\alpha 10 \times \mathrm{H}+\beta 10 \times \mathrm{V} \\
& \text { leadV5 }=\alpha 11 \times \mathrm{H}+\beta 11 \times \mathrm{V} \\
& \text { leadV6 }=\alpha 12 \times \mathrm{H}+\beta 12 \times \mathrm{V}
\end{aligned}
$$

in which $\mathrm{H}$ is the horizontal vector, $\mathrm{V}$ is the vertical vector and $\alpha 1 \ldots \alpha 12$ and $\beta 1 \ldots \beta 12$ are the transformation coefficients. Leads III, aVR, aVL, and aVF are redundant and are calculated from known geometries in the Einthoven triangle. 


\subsubsection{APPLICATION OF MATRIX TO VALIDATION DATASET}

The measured ECG data for the validation dataset were imported into MATLAB (a program for data processing, using the eHSP library where QRS complexes were automatically identified for all beats in the signal. The starting position of the $\mathrm{P}$ wave was stored into an array. Each lead signal was then filtered in MATLAB using a 1st order high pass filter. For each starting position element in the array the next 46 samples were collected to ensure that the entire signal of interest was identified. Once all beats were acquired in this way for each lead, these were averaged for each lead, resampled from 1000 to $256 \mathrm{~Hz}$ and stored. This produced an averaged beat for each lead and each patient of 22 samples. The averaged beats from study leads $\mathrm{H}$ and $\mathrm{V}$ were then combined with the transformation matrix to generate eight independent derived beats (lead I, II, V1-V6).

The derived leads were compared to the measured leads for each patient in RAW format. Continuous data were presented as mean and standard deviation and categorical variables were presented as frequencies and percentages. The quantitative measures of similarity between the original (measured) ECG and the corresponding derived (reconstructed) ECG were determined using Pearson $r$ correlation and root mean square error (RMSE) analysis for each derived lead. The Pearson $r$ was considered to show high positive correlation at $r \geq 0.7$. The RMSE is a parameter that indicates the average voltage error (microvolts) across the ECG leads studied. These parameters have been used by other investigators who have recorded this type of data for derived ECG leads (Astar, 2009).

From the setup, a sensor-system that is appropriate for self-care is proposed with an evaluation system by performing a series of experiments using patient simulators setup to perform a function for elderly persons. The results of ECG comparisons in terms of correlation, RMS values, as well as changes in diagnosis probability and of selected ECG measurements were experimented with aim to supporting the scenario of self-care in cardiology. The following case scenario was experimented:

a) Simulated electrodes in their standard locations, so that analysis can be obtained using the current knowledge background and already applicable algorithms in order to process the signal and to provide a digital interpretation.

b) Simulated and recorded a 3-lead (I, II and V2) ECG in order to detect cardiovascular diseases

c) Simulated a sensor system that can be put on and off very easily, so that the user can discretely check own health status anywhere and at any time.

d) Enabled compatibility simulation to the morphology of each sensor-system user in order to obtain a diagnostic quality ECG signal.

\section{RESUltS}

The ECG signal was measured through e-health sensor shield and Raspberry Pi picked from a patient simulator and transferred to the MATLAB environment. To demonstrate the quality of the data that can be collected from the eHSP, the ECG results are displayed in Table 1. 
International Journal of Biomedical Engineering and Science (IJBES), Vol. 5, No. 3/4, October 2018

Table 1. Pearson correlation coefficients (r) for all patients and leads

$\begin{array}{lll}\text { Lead } & \text { RMSE } & \text { Pearson } \boldsymbol{r} \text { in } \boldsymbol{\mu V} \\ \text { I } & 292.6 & 0.585 \\ \text { II } & 275.1 & 0.8579 \\ \text { V1 } & 359.58 & 0.8413 \\ \text { V2 } & 643.93 & 0.719 \\ \text { V3 } & 638.76 & 0.6802 \\ \text { V4 } & 618.2 & 0.5989 \\ \text { V5 } & 455.88 & 0.7287 \\ \text { V6 } & 242.99 & 0.8841\end{array}$

$\begin{array}{lllllllll}\text { Case } & \text { I } & \text { II } & \text { V1 } & \text { V2 } & \text { V3 } & \text { V4 } & \text { V5 } & \text { V6 } \\ \mathbf{1} & 0.463 & 0.945 & 0.984 & 0.664 & 0.738 & 0.817 & 0.903 & 0.944 \\ \mathbf{2} & 0.661 & 0.985 & -0.442 & 0.771 & 0.948 & 0.975 & 0.980 & 0.984 \\ \mathbf{3} & -0.440 & 0.847 & 0.981 & 0.949 & 0.949 & 0.930 & 0.859 & 0.554 \\ \mathbf{4} & 0.966 & 0.774 & 0.989 & 0.992 & 0.924 & 0.574 & 0.692 & 0.707 \\ \mathbf{5} & 0.738 & 0.947 & 0.960 & 0.837 & 0.768 & 0.597 & 0.960 & 0.967 \\ \mathbf{6} & 0.970 & 0.988 & 0.217 & -0.223 & 0.847 & 0.971 & 0.987 & 0.995 \\ \mathbf{7} & 0.919 & 0.644 & 0.996 & 0.995 & 0.971 & 0.575 & 0.990 & 0.994 \\ \mathbf{8} & 0.921 & 0.982 & 0.957 & 0.860 & 0.636 & 0.507 & 0.973 & 0.997 \\ \mathbf{9} & 0.607 & 0.881 & 0.993 & 0.964 & 0.938 & 0.896 & 0.866 & 0.949 \\ \mathbf{1 0} & 0.735 & 0.954 & 0.992 & 0.919 & 0.832 & 0.862 & 0.986 & 0.991 \\ \mathbf{1 1} & 0.810 & 0.944 & 0.979 & 0.940 & 0.935 & -0.230 & 0.975 & 0.994 \\ \mathbf{1 2} & -0.137 & 0.849 & 0.974 & 0.963 & 0.949 & 0.913 & 0.793 & 0.288 \\ \mathbf{1 3} & 0.944 & 0.885 & 0.995 & 0.990 & 0.959 & 0.952 & 0.974 & 0.971 \\ \mathbf{1 4} & 0.934 & 0.988 & 0.918 & 0.844 & 0.948 & 0.954 & 0.976 & 0.989 \\ \mathbf{1 5} & 0.774 & 0.969 & 0.846 & 0.382 & 0.865 & 0.951 & 0.929 & 0.943 \\ \mathbf{1 6} & 0.905 & 0.984 & 0.865 & 0.152 & 0.743 & 0.960 & 0.982 & 0.993 \\ \mathbf{1 7} & 0.917 & 0.989 & 0.985 & 0.952 & 0.819 & 0.754 & 0.977 & 0.983 \\ \mathbf{1 8} & 0.546 & 0.948 & 0.974 & 0.980 & 0.995 & 0.992 & 0.781 & 0.595 \\ \mathbf{1 9} & 0.968 & -0.280 & 0.969 & 0.969 & 0.942 & 0.866 & 0.755 & 0.932 \\ \mathbf{2 0} & 0.815 & 0.885 & 0.768 & 0.427 & 0.637 & 0.849 & 0.891 & 0.924 \\ \mathbf{2 1} & 0.809 & 0.723 & 0.960 & 0.962 & 0.961 & 0.710 & 0.779 & 0.973 \\ \mathbf{2 2} & 0.598 & 0.939 & 0.922 & 0.510 & 0.168 & 0.507 & 0.909 & 0.967 \\ \mathbf{2 3} & 0.626 & 0.963 & 0.777 & 0.105 & 0.347 & -0.063 & -0.094 & 0.348 \\ \mathbf{2 4} & 0.846 & 0.977 & 0.985 & 0.851 & 0.721 & 0.946 & 0.935 & 0.966 \\ \mathbf{2 5} & -0.302 & 0.994 & -0.092 & -0.376 & 0.817 & 0.848 & 0.903 & 0.963 \\ \mathbf{2 6} & -0.598 & 0.991 & 0.924 & 0.319 & -0.406 & -0.617 & -0.470 & 0.971 \\ \mathbf{2 7} & -0.073 & 0.935 & 0.926 & 0.973 & 0.981 & 0.973 & 0.901 & 0.949\end{array}$




\section{DISCUSSIONS}

The data extracted from the cloud database is separated by comma value in csv format as the processing was conducted. This data underwent a series of known ECG processing procedures to extract desired features. The eHSP ECG sensor consists of the E, M and Ground electrodes. The $\mathrm{E}$ and $\mathrm{M}$ electrodes leads monitor the aggregated electrical activity of all the muscles underneath the electrodes and the ground provides a reference point.

The results showed a significant correlation (Table 1) between heart rate measured by eHSP ECG and by Polar MS4000 with correlation coefficients ranging from 0.97 to 1.00 . In test 1 the mean difference \pm 2 SD between heart rate Polar and heart rate ECG was $0.7 \pm 4.3 \mathrm{bpm}$ and in test 2, $0.2 \pm 3.2 \mathrm{bpm}$. In the repeated tests, the mean difference of heart rate between test 2 and test $1 \pm 2 \mathrm{SD}$ was $3.2 \pm 11.9 \mathrm{bpm}$ with eHSP ECG and 2.6 $\pm 14.3 \mathrm{bpm}$ with Polar RS400 and these differences were not statistically significant as shown in Figure 3.
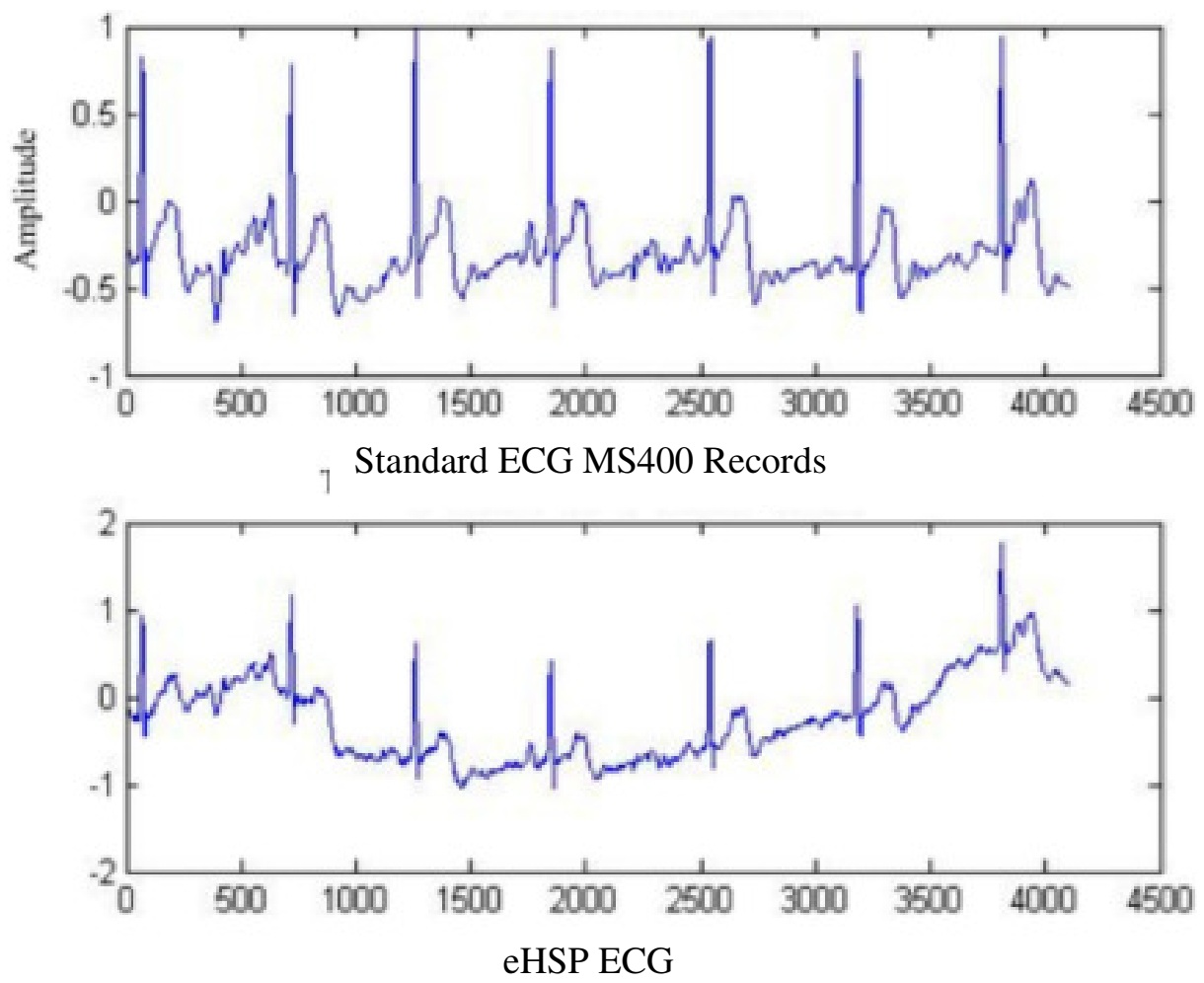

Figure 1: ECG Repeatability results

This study indicates good criterion-related validity and test-retest repeatability of Polar MS400 and eHSP ECG. Differences observed at individual position levels should be noticed but are not considered to be significantly important. The eHSP ECG is thus well suited for recording heart rate at any location for monitoring the heart condition of mobile person. 


\section{Proposed Model Design}

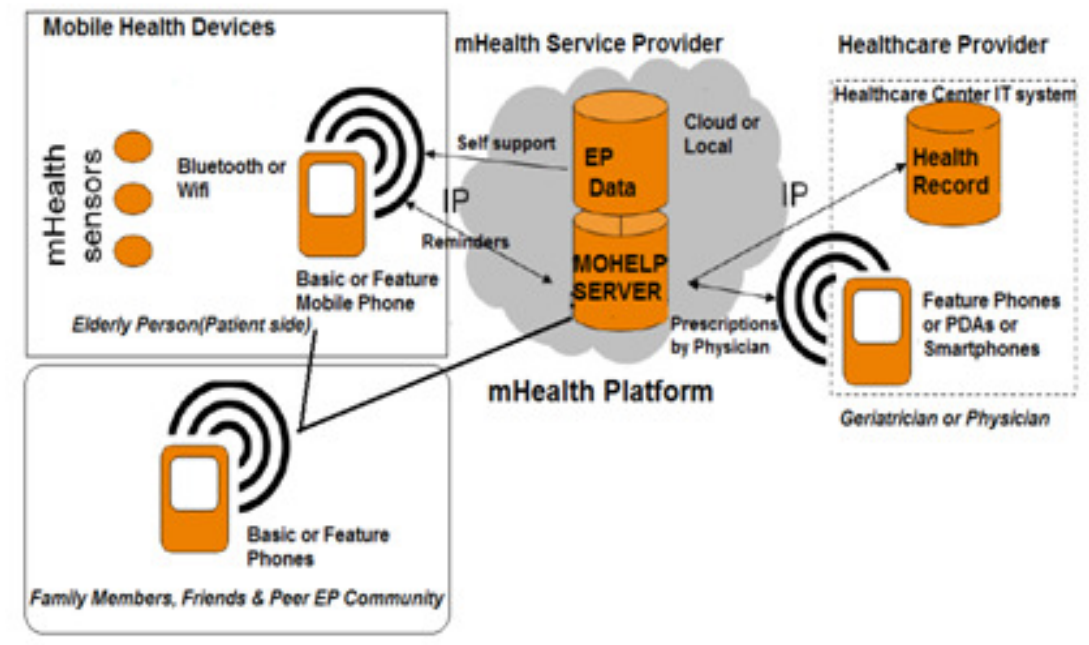

Figure 4: Proposed components of MOHELP Model Based on eHSP

The address the above questions raised in, we propose a sensor based mobile health referred herein as the "Mobile Health Service for the ELderly Persons (MOHELP)" for telecare. The proposed framework is divided into building blocks and sub-building blocks to fit the sociotechnical needs of users and telecare providers in a pervasive environment. Figure 4 shows the building blocks and sub-building blocks. The building blocks show eHSP applied with e.g. ECG bio-sensors, cellphones/tablets etc., the mobile Health system linked by mobile networks to Healthcare providers and Healthcare Centers.

Many of the building blocks inside the generic architecture and eHSP do not show the basic functionality that a generic mobile health Service will need. The functionalities are service flow processes such as diagnosis, treatment alerts, monitoring vital signs, consultation communications and or appointments etc. However, the exact details of the functionality and its architecture can be determined through requirement collections from the biosensors, in this case ECG. However, more sensors of the eHSP can be applied simultaneously.

The proposed framework is a hosted Bio-Wearable Health Information Technology (BWHIT) framework that uses the data generated by the mobile networks to provide an enhanced healthcare service over traditional tele-health offerings. The architecture of the proposed HIT framework is provided in Figure 5. The proposed architecture has user interfaces, with a middleware acting as a broker between various bio-wearable eHSP use cases that will deployed into a server. The hosted BWHIT system can be setup at a Hospital facility, or in the clouds. 


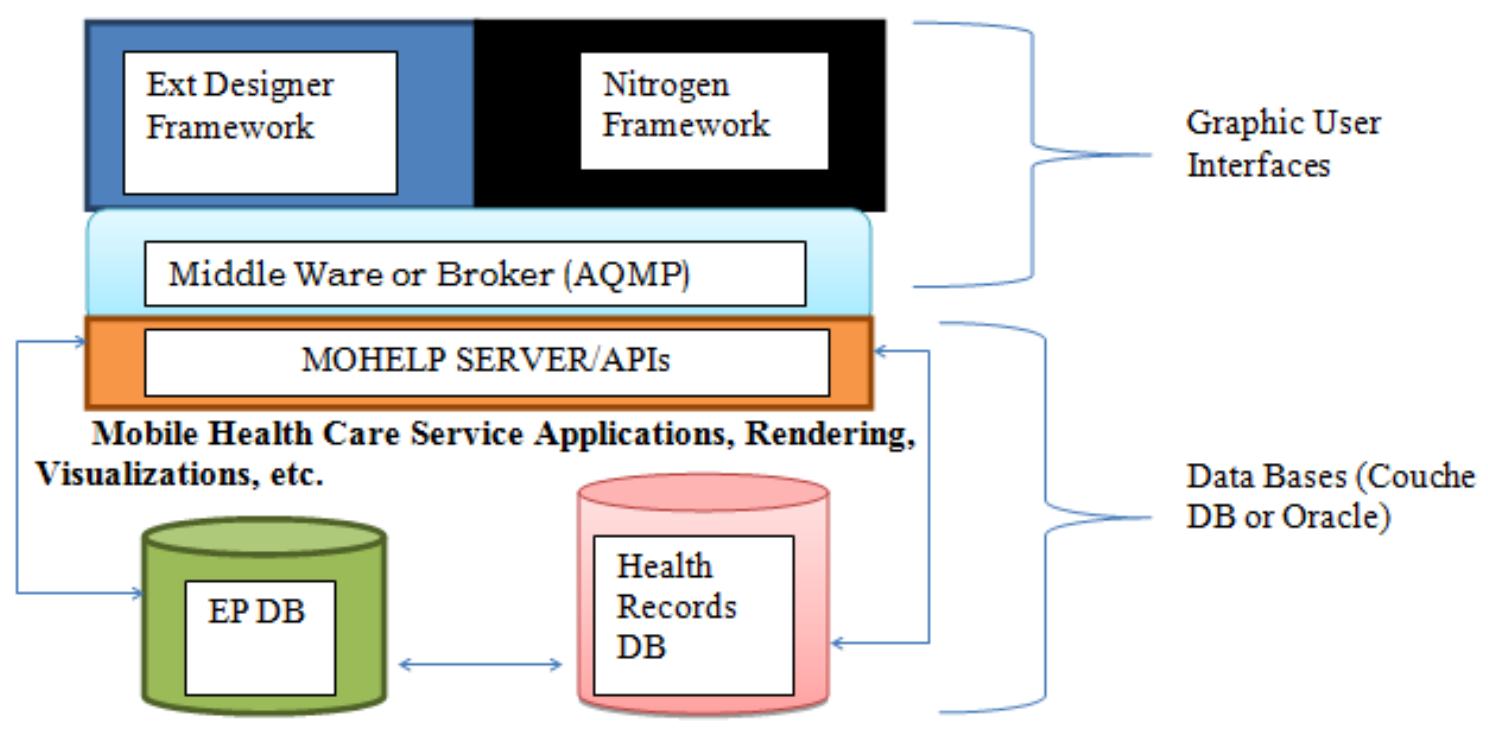

Figure 5: The eHSP Architecture for the elderly for Non-invasive Monitoring

\section{CONCLUSION}

The application of biosensor networks shows that a new generation of technology which can be created for patient monitoring with minimal cost, with the possibility of reducing the number of occupied beds in hospitals and improving health workers performance. In addition, applying various methodologies and design of a system architecture in software terns for mining ECG from a patient for diagnosis to be monitored continuously anywhere and anytime has been hypothetically achieved in the study. The portable size of the eHSP and its ability to assimilate various exclusive sensors permits non-invasive monitoring of different biosignals outside of a medical facility or environment. The system has the additional capability of shifting patient data directly to a cloud database using a mobile device. With the DES method an EDA framework was created to transfer Bio-signal data collected in various scenarios with no Internet connectivity and data stored on the mobile devices such as android smart phones hard drive using blue tooth.

\section{FUTURE WORKS}

This research study has a distinction of taking bits from various research domains, for example biosensors in biomedical engineering, health sciences and software engineering. Therefore, the contribution it provides tends to influence several domains and so to trigger the prospective research work routes at the application level as well as at the conceptual level. At the application level, only one biosensor of eHSP was used with hypothetical data simulated from a patient simulator. The patients' morphological data collected using the patient simulator did not allow taking the data directly. The biosensor system applied directs to provide decisions based on patients' historical data, real-time data gathering, and thus eliminating simulated data remains a consideration. Further works to enhance the functionality of the system by adding more sensors and using it to collect data from a larger sample size of patient needs also to be considered. Finally, the designed framework for event driven data health services transposition can be 
extended to other domains, for instance to support e-autonomous resources management system. A comparison and consequently the improvement of a set of intelligent biosensor systems would provide an evidence-based data set that would be used to the enhance autonomous self-care knowledge.

\section{REFERENCES}

[1] Akter, Shahriar, D'Ambra, John, Ray, Pradeep, (2010). User Perceived Service Quality of m-Health Services in Developing Countries. 18th European Conference on Information Systems. Available at: http://is2.lse.ac.uk/asp/aspecis/20100030.pdf. Accessed in January, 2016.

[2] Anderson., (2009). Mobile Health Application for the Health Sector.ICT Sector Unit: World Bank. 1818 H Street NW, MSN MC6-616, Washington DC 20433, USA. Available at: myamamichi@worldbank.org. Retrieved on 28thJanuary, 2012.

[3] ANSI', 2001, ANSI. Diagnostic electrocardiographic devices (ANSI/AAMI EC11:1991/(R)2001). Arlington, VA: Association for the Advancement of Medical Instrumentation, 2000, p.

[4] Asta. (2011). Participatory action research and action science compared: A commentary. American Behavioral Scientist, 32(5), 612-623.

[5] Bonner R.E., Crevasse L., Ferrer M.I., et al.(1972). A new computer program for analysis of scalar electrocardiograms. Computers and Biomedical Research, vol. 5, no. 6, pp. 629-653.

[6] Borromeo S., Rodriguez-Sanchez C., Machado F., et al., (2007). A reconfigurable, wearable, wireless ECG system. In: Proc. of 29th annual intern. conf. on Engineering in Medicine and Biology Society (EMBC), 2007, Lyon, France: IEEE, pp. 1659-1662.

[7] Bortolan G., Willems J.L., (1993). Diagnostic ECG classification based on neural networks. Journal of Electrocardiology, 1993, suppl. 26, pp. 75-79.

[8] British Standard, (2003). Medical electrical equipment. Particular requirements for safety. Particular requirements for safety, including essential performance, of recording and analysing single channel and multichannel electrocardiographs. BS EN 60601-2-51: BSI, 2003, 86 p. ISBN 0580427285

[9] British Standard. Size Designation of Clothes. Measurements and intervals. BS EN 13402-3: BSI, 2004, 32 p. ISBN 0580 455718. [Brodnick'00], Brodnick D. A method to locate electrode placement. Journal of Electrocardiology, 2000, suppl. 33, pp. 211-218.

[10] Callens L., Carrault G., Cordier M.O., et al. Intelligent adaptive monitoring for cardiac surveillance. In. Proc. of the 18th European conf. on Artificial Intelligence, 2008, Patras, Greece: IOS Press, pp. 653-657.

[11] Castelli G., Mamei M., Zambonelli F. Engineering contextual knowledge for autonomic pervasive services. Journal of Information and Software Technology, 2008, vol. 50, no. 1, pp. 36-50.

[12] Cauvet C., Guzelian G., (2008). Business Process Modeling: A Service-Oriented Approach. In: Proc. of the 41st annual intern. conf. on System Sciences (HICSS2008), 2008, Waikoloa, HI, USA: IEEE, pp. 98-98.

[13] Cornfield J., Dunn R.A., Batchlor C.D., et al., (1973) Multigroup diagnosis of electrocardiograms. Computers and Biomedical Research, 1973, vol. 6, no. 1, pp. 97-120.

[14] Czarnecki Krysztof, Eisenecker Ulrich., (2000). Generative Programming: Methods, Tools, and Applications. 1st ed.: Addison-Wesley Professional, 2000, 864 p. ISBN 9780201309775

[15] Cooking Hacks., (2012). e-Health Sensor Platform Complete Kit V2.0 for Arduino, Raspberry Pi and Intel Galileo [Biometric / Medical Applications: available at: https://www.cookinghacks.com/ehealth-sensors-complete-kit-biometric-medical-arduino-raspberry-pi

[16] Darrell W., (2015). Issues in Technology innovation Vol. 9.Center for Technology innovations at Brookings. Available at: xx Retrieved on 2nd February, 2012.

[17] Debra (2012). Will physicians trust mobile health data? Available at: http://www.diversinet.com/PDFS/news/2010/Will_\%20physicians_trust_mobile_health_data.pdf. Retrieved on 4thMarch, 2012. 
[18] De Luca G., Suryapranata H., Ottervanger J.P., et al., (2004). Time Delay to Treatment and Mortality in Primary Angioplasty for Acute Myocardial Infarction. Circulation, 2004, vol. 109, no. 10, pp. 1223 -1225 .

[19] Debenham John K., (1989). Knowledge Systems Design. Australia: Prentice-Hall, 1989, 583 p. ISBN 9780135171295

[20] Di Rienzo M., Rizzo F., Meriggi P., et al., (2007). MagIC: a textile system for vital signs monitoring. Advancement in design and embedded intelligence for daily life applications. In: Proc. of the 29th annual intern. conf. on Engineering in Medicine and Biology Society (EMBS), 2007, Lyon, France: IEEE, pp. 3958-3961.

[21] Donnelly M., Nugent C.D., Finlay D., et al., (1984). Optimal electrode placement for the identification of old MI and LVH. In: Proc. of the 33rd intern. conf. on Computers in Cardiology, 2006, Valencia, Spain: IEEE, pp. 437-440. ISBN 9781424425327.

[22] Dower G.E., (1984). The ECGD: a derivation of the ECG from VCG leads. J Electrocardiol, 1984, vol. 17, no. 2, pp. 189-191

[23] Enrique, (2010). Usability, Accessibility, Human Factors \& Design: Available at: http://www.mhimss.org/resource-topics/usability-accessibility-human-factors-design: Retrieved on 8th May, 2012.

[24] Ezati Marino, Vander Hoorn S, Rodgers A et al. (2006) Estimates of global and regional potential health gains from reducing multiple major risk factors. Lancet 362, 271-280.

[25] Fayn J. A, (2011). Classification Tree Approach for Cardiac Ischemia Detection Using Spatiotemporal Information From Three Standard ECG Leads. IEEE Trans Biomed Eng, 2011, vol. 58, no. 1, pp. 95-102.

[26] Fayn J., Macfarlane P.W., Rubel P., (2007). Can the lessons learned from the assessment of automated electrocardiogram analysis in the Common Standards for quantitative Electrocardiology study benefit measurement of delayed contrast-enhanced magnetic resonance images? J Electrocardiol, 2007, vol. 40, no. 3, pp. 246-250.

[27] Fayn J., Rubel P., (1988). CAVIAR: a serial ECG processing system for the comparative analysis of VCGs and their interpretation with auto-reference to the patient. Journal of Electrocardiology, 1988, vol. 21, pp. 173-176.

[28] Fayn J., Rubel P., Pahlm O., et al., (2007). Improvement of the detection of myocardial ischemia thanks to information technologies. Int J Cardiol, vol. 120, no. 2, pp. 172-80.

[29] Fayn J., Rubel P., (2010). Toward a Personal Health Society in Cardiology. IEEE Transactions on Information Technology in Biomedicine: A Publication of the IEEE Engineering in Medicine and Biology Society, vol. 14, pp. 409-409.

[30] Fahy C., Davy S., Boudjemil SZ., et al., (2008). Towards an Information Model That Supports Service-Aware, Self-Managing Virtual Resources. In: van der Meer S., Burgess M., Denazi S.G. Eds. Lecture Notes in Computer Science on Modelling Autonomic Communications Environment, Proc. of the 3rd intern. workshop on Modelling Autonomic Communications Environments (MACE2008), Samos Island, Greece: Springer, pp. 102-107.

[31] Finlay D., Nugent C.D., Donnelly M.P., et al., (2008). Optimal electrocardiographic lead systems: practical scenarios in smart clothing and wearable health systems. IEEE Transactions on Information Technology in Biomedicine, 2008, vol. 12, no. 4, pp. 433-41.

[32] GSMA, (2009-2011). Mobile Technology's Promise for Healthcare: Available at: http://rotarykilifi51224.blogspot.com/2012/02/mobile-health-mhealth-project-improving.html. Retrieved on 8thMarch, 2012.

[33] GSMA, (2012). Touching lives through mobile health Assessment of the global market opportunity. Available at: www.pwc.com/in/en/assets/.../gsma-pwc_mhealth_report.pdf. Retrieved on 3rdFebruary, 2012.

[34] GSMA, (2013). M-Health Technologies: Applications to Benefit Older Adults. Position Paper.

[35] Ghoreishi S. F. and Allaire D. L. (2017). Adaptive Uncertainty Propagation for Coupled Multidisciplinary Systems", AIAA Journal, Vol. 55, No. 11 (2017), pp. 3940-3950 
International Journal of Biomedical Engineering and Science (IJBES), Vol. 5, No. 3/4, October 2018

[36] Mahdi and Ulisses R. (2017). Particle Filters for Partially-Observed Boolean Dynamical Systems: Article in Automatica 87:238-250 • January 2018

[37] Micheal, (2014). "M-healthcare for patient self-management: a case for diabetics." Int. J. Electron. Healthc. 2004; 1(2):221-241.

[38] McCann, et al. (2007). "Networks of Action: Sustainable Health Information

[39] Pantley H. (2014). Patient adherence to medical treatment: a meta review. ISBN 90-6905-7980.

[40] Pollen J.H., (2011). CMMI for Services: Guidelines for Superior Service, 2nd ed.: Addison-esley, 2011, 800 p. ISBN 0-321-71152-Systems Across Developing Countries." MIS Quarterly 28(3): 337362

[41] Shailian, Xie et al. (2018). Reconstruction of the standard 12-lead ECG from recordings using nonstandard activity-compatible proximal limb lead positions. Journal of Electrocardiology, 1995, vol. 28, no. 1, pp. 33-38.

[42] Rajaganeshan. (2008). Service coordination and integration in a developmental systems approach to early intervention. In M. J. Guralnick (Ed.), The developmental systems approach to early intervention (pp. 29-58). Baltimore: Brookes

[43] Rubel J.M, (2005). Situation awareness: In search of meaning. CSERIAC Gateway, 1996, vol. 6, no. 6, pp. 1-4.

[44] Sejersten G., (2008). Improved EASI coefficients: their derivation, values, and performance. Journal of Electrocardiology, 2002, vol. 35, pp. 23-33.

[45] Schijveneers., (1997). Improved EASI coefficients: their derivation, values, and performance. Journal of Electrocardiology, 2002, vol. 35, pp. 23-33..

[46] WHO (2008). Aging in Sub-Saharan Africa: Recommendation for Furthering Research. National Research Council (US) Committee on Population. National Academies Press (US), Washington (DC).

[47] WHO, (2010-2015). Technologies to Help Older Adults Maintain Independence: Advancing Technology Adoption', Center for Technology and Aging

[48] WHO (2012) A Minimum Data Set on Ageing and Older Persons in Sub-Saharan Africa: Process and Outcome. African Population Studies 21, 19-36.

[49] WHO, Frederick Nyanzi, (2008). Case Study on the Older Persons of Uganda. The Aged Family Uganda (TAFU). Available at: www.tafu.org/PDF/Concept_Paper.pdf. Accessed in February, 2012.

[50] Zipes. (2006). Using Ontologies for Simulation Modeling. In: Proc. of the 38th conf. on Winter Simulation (WSC'06), 2006, Monterey, CA, USA: IEEE Press, pp. 1151-1159 